\section{Índice de Desenvolvimento Humano e prevenção secundária de câncer de mama e colo do útero: um estudo ecológico}

\author{
Human Development Index and secondary \\ prevention of breast and cervical cancer: \\ an ecological study \\ Índice de Desarrollo Humano y prevención \\ secundaria del cáncer de mama y cáncer \\ de cuello uterino: un estudio ecológico
}

\begin{abstract}
This ecological study assessed mammography and Papanicolaou test (Pap smear) screening rates and their association with the Human Development Index (HDI) in Brazilian state capitals and Federal District in 2011, based on VIGITEL telephone survey data. More than $70 \%$ of women had a mammogram some time in life in all the state capitals, while the Pap smear rates any time in life and in the previous three years fell short of the $80 \%$ target in most state capitals. There was a strong positive correlation between HDI and the test rates ( $r=0.52$ and 0.66 for mammogram any time in life and in the previous two years and $r=0.66$ and 0.71 for Pap smear any time in life and in the previous three years, respectively). Prevalence ratio (PR) for mammogram in the previous two years was 1.06 (95\% CI: 1.01-1.10) and PR for Pap smear in the previous three years was 1.07 (IC95\%: 1.041.10). The promotion of public health policies that encourage Brazil's urban economic and social development should help increase coverage for these tests.
\end{abstract}

Papanicolaou Test; Mammography; Human Development; Secondary Prevention
Ana Daniela Izoton de Sadovsky 1,2 Wanêssa Lacerda Poton 2,3 Bárbara Reis-Santos 2

Mara Rejane Barroso Barcelos 1,2 Inacio Crochemore Mohnsam da Silva ${ }^{2}$

\section{Resumo}

Estudo ecológico que analisou a proporção da realização de mamografia e do exame de Papanicolaou e a associação destes com o Índice de Desenvolvimento Humano (IDH), nas 26 capitais brasileiras e Distrito Federal, em 2011, com dados do VIGITEL. A proporção de mamografia em algum momento da vida esteve acima de $70 \%$ para todas as capitais estudadas. Entretanto, poucas capitais atingiram a meta de $80 \%$ para o Papanicolaou, tanto para a realização em algum momento da vida como nos últimos três anos. Houve forte correlação positiva entre IDHe as proporções desses exames $(r=0,52$ e 0,66 para realização de mamografia em algum momento da vida e nos últimos dois anos e $r=0,66$ e 0,71 para realização do Papanicolaou em algum momento da vida e nos últimos três anos, respectivamente). A razão de prevalência de mamografia nos últimos dois anos foi 1,06 (IC95\%: 1,01-1,10) e para o Papanicolaou nos últimos três anos foi 1,07 (IC95\%: 1,04-1,10). A promoção de políticas públicas que incentivem o desenvolvimento socioeconômico das cidades brasileiras favoreceria o aumento da cobertura desses exames.

Teste de Papanicolaou; Mamografia; Desenvolvimento Humano; Prevenção Secundária 


\section{Introdução}

O Instituto Nacional de Câncer José Alencar Gomes da Silva (INCA) destaca o câncer de mama e de colo do útero como primeiro e quarto lugares, respectivamente, entre as principais neoplasias que acometem as mulheres em países desenvolvidos e, em primeiro e terceiro, nas mulheres brasileiras 1. A detecção precoce do câncer de mama pode ser realizada por meio da mamografia e, do câncer de colo do útero, pelo exame de Papanicolaou. A sobrevida média das mulheres com câncer de mama, após cinco anos do diagnóstico, é de cerca de $85 \%$ nos países de renda alta, e em torno de $60 \%$ nos países com renda média e baixa 1. A detecção precoce dessas morbidades é fundamental para um bom prognóstico e consiste na principal estratégia de prevenção secundária 1,2,3.

A Organização Mundial da Saúde (OMS) e o Ministério da Saúde preconizam que $70 \%$ das mulheres com 50 a 69 anos realizem uma mamografia a cada dois anos e $80 \%$, de 25 a 64 anos, o exame de Papanicolaou a cada três anos 2,3. Em 2008, a mamografia no Brasil atingiu uma cobertura de $71,5 \%$ nessas mulheres ${ }^{4}$ e o exame de Papanicolaou foi realizado em 87,6\% 5 . Apesar da tendência crescente na realização desses exames na população alvo, estudos recentes têm demonstrado que essa cobertura ainda é baixa nas mulheres com maior vulnerabilidade social, intimamente relacionada à pobreza e baixa escolaridade na população 6,7,8,9. Assim, é de suma importância a compreensão dos determinantes sociais que podem estar associados à disponibilização e à realização da mamografia e do exame de Papanicolaou, para identificação de grupos populacionais com menor acesso a esses exames e elaboração de estratégias que combatam essas iniquidades.

Indicadores socioeconômicos, individuais e regionais, são preditores independentes de incidência, diagnóstico, tratamento e prognóstico dos diversos tipos de câncer bem reconhecidos e documentados 10 .

O Índice de Desenvolvimento Humano (IDH) é um indicador que descreve as características socioeconômicas, sendo composto por dados sobre a expectativa de vida ao nascer, a educação e o Produto Interno Bruto per capita 11. Ele está inversamente associado com as taxas de incidência de câncer no geral 12 e, quando se considera os tipos de câncer individualmente, esta associação se mantém para o câncer de colo de útero, mas torna-se diretamente associada com as taxas de câncer de mama ${ }^{13}$. Considerando a importância da incidência da doença na organização dos sistemas de saúde para ofertas de serviços 13 e a incipiente evidência que considera esse indicador como componente coletivo para a compreensão das disparidades em saúde, este estudo teve como objetivo analisar a proporção da realização da mamografia e do exame de Papanicolaou e a associação destes exames com o IDH nas capitais brasileiras e no Distrito Federal no ano de 2011.

\section{Métodos}

Foi realizado um estudo ecológico utilizando como unidade amostral as capitais brasileiras e o Distrito Federal com base nos dados secundários obtidos do VIGITEL (Vigilância de Fatores de Risco e Proteção para Doenças Crônicas por Inquérito Telefônico) no ano de 2011 14. O VIGITEL é um inquérito telefônico que foi implantado em 2006 nas 26 capitais dos estados brasileiros e no Distrito Federal, com o objetivo de monitorar os principais fatores determinantes condicionantes das doenças e agravos crônicos não transmissíveis no Brasil. Ele avalia anualmente cerca de 2.000 indivíduos maiores de 18 anos em cada capital e Distrito Federal. O processo de amostragem é baseado no cadastro de linhas das empresas de telefonia fixa, realizando a seleção da amostra de forma aleatória e estratificada em cada capital. Em 2011, o VIGITEL realizou ligações para 111.200 linhas telefônicas elegíveis para o estudo. O percentual de recusas foi de $2,2 \%$ das linhas elegíveis, sendo necessárias em torno de 13 ligações para cada entrevista completa. O detalhamento dos métodos empregados no estudo pode ser obtido nas publicações do Ministério da Saúde 14.

Baseado nas recomendações da OMS e do Ministério da Saúde para o diagnóstico precoce do câncer de mama e do colo do útero, os desfechos do presente trabalho foram os seguintes indicadores: (1) proporção de mulheres que realizaram mamografia (i) em algum momento de sua vida e (ii) nos últimos dois anos e (2) proporção de mulheres que realizaram exame de $\mathrm{Pa}$ panicolaou (i) em algum momento de sua vida e (ii) nos últimos três anos para cada capital e Distrito Federal. Para avaliação da realização da mamografia, este estudo incluiu apenas a faixa etária de risco entre 50 e 69 anos 15. Em relação à realização do exame de Papanicolaou, os dados registrados no VIGITEL foram de mulheres de 25 até 59 anos, apesar da nova recomendação do Ministério da Saúde que ampliou recentemente a faixa etária de risco para 25 a 64 anos 16 .

O IDH, principal exposição estudada, é um indicador que varia de 0 (nenhum desenvolvimento humano) a 1 (desenvolvimento humano 
total) e considera-se baixo o IDH nos municípios entre 0,000-0,499, médio de 0,500-0,799 e alto entre 0,800-1,000 11. O IDH de cada capital e do Distrito Federal foi obtido no Instituto Brasileiro de Geografia e Estatística (IBGE), estimados por meio de dados do Censo Demográfico de 201011.

Outras variáveis relativas às capitais brasileiras incluídas nas análises foram extraídas da base de dados do VIGITEL, dentre elas: idade média (anos), escolaridade média (anos de estudos), proporção de mulheres com cor da pele declarada branca, proporção de solteiras e prevalência de tabagismo.

Valendo-se do Sistema de Informação da Atenção Básica (SIAB) foram extraídas informações sobre a cobertura da Estratégia Saúde da Família (ESF) e, com base no Instituto de Pesquisa Econômica e Aplicada (IPEA), o Índice de Gini no ano de 2010. Esse indicador mede o grau de desigualdade econômica no rendimento domiciliar per capita, em que o valor de 0 representa igualdade perfeita e um, desigualdade extrema (IPEA; http://www.ipeadata.gov.br/, acessado em 05/Abr/2014).

Os fatores de ponderação foram empregados nas análises para equilibrar as diferenças amostrais 17 , buscando a representatividade de todas as capitais brasileiras e não apenas da população que possuía linha telefônica. Cabe salientar que os procedimentos de ponderação utilizados neste trabalho foram os mesmos usados em publicações do VIGITEL baseando-se no ano de 2012, levando em consideração as estimativas atualizadas da população de acordo com o censo de 2010 17. Assim, os resultados referentes à proporção de realização dos exames estudados podem diferir do relatório publicado referente ao ano de 201114.

As médias e prevalências populacionais foram apresentadas em gráficos e tabelas. Os gráficos de proporção da realização dos desfechos estudados foram construídos confrontando-os com a meta preconizada pelo Ministério da Saúde de $70 \%$ e $80 \%$ para realização de mamografia e de teste de Papanicolaou, respectivamente. Estimaram-se os coeficientes de correlação de Pearson para analisar a correlação entre a exposição principal de interesse e os desfechos estudados; para a exposição principal e as demais covariáveis, com o objetivo de definir aquelas que seriam incluídas nos modelos ajustados de regressão. Para verificar as associações foram construídos modelos brutos e ajustados de regressão de Poisson com variância robusta, cuja variável dependente foi proporção de realização de exames e, a variável independente, o IDH com distribuição padronizada devido ao seu intervalo de valores entre zero e um. No primeiro modelo ajustado foram incluídas as covariáveis relacionadas com a determinação individual da realização dos exames identificados em estudos prévios (idade média, estado civil, tabagismo) 6,7,8,9 e nos dois últimos modelos foram incluídos o Índice de Gini e a cobertura de ESF 18 relacionados com a determinação social e programática, respectivamente. As análises foram realizadas no programa Stata 12 (Stata Corp., College Station, Estados Unidos).

O projeto VIGITEL foi aprovado pela Comissão Nacional de Ética em Pesquisa (CONEP) para seres humanos do Ministério da Saúde e a base de dados é de domínio público.

\section{Resultados}

Em 2011, o VIGITEL realizou 54.144 entrevistas completas nas 26 capitais brasileiras e no Distrito Federal. Entre as principais características da população estudada evidencia-se que 31.503 foram mulheres, as medianas de idade e de escolaridade foram 49 e 10 anos, respectivamente, e nas capitais dos estados que compõem a Região Sul (Curitiba, Florianópolis e Porto Alegre) mais de $50 \%$ se declararam como de cor branca. Outras características dessa população estão na Tabela 1.

Em relação aos indicadores socioeconômicos (Tabela 2), observou-se que Maceió $(0,721)$, Rio Branco $(0,727)$, Macapá $(0,733)$, Porto Velho $(0,736)$ e Belém $(0,746)$ foram as capitais que apresentaram os menores IDH. Quanto ao Índice de Gini, aquelas que apresentaram os índices mais baixos foram Boa Vista $(0,58)$, Belém $(0,61)$, Rio Branco $(0,59)$ e Belo Horizonte $(0,60)$. A cobertura da ESF foi um indicador com ampla variação entre as capitais estudadas, desde Teresina apresentando $98 \%$ de cobertura até o Distrito Federal com 15\% (Tabela 2).

A proporção de realização do exame de mamografia em algum momento da vida esteve acima dos $70 \%$ para todas as capitais estudadas (Figura 1a). A proporção de realização desse exame nos últimos dois anos foi baixa em Rio Branco (54\%; IC95\%: 44-63) e Fortaleza (63\%; IC95\%: 57 69); apesar dos intervalos de confiança em Belém (63\%; IC95\%: 54-71), Macapá (66\%; IC95\%: 52-81) e Manaus (69\%; IC95\%: 62-77) contemplarem valores preconizados pelo Ministério da Saúde, suas médias foram abaixo do esperado (Figura 1b).

Em relação à proporção de realização do exame de Papanicolaou foram dez as capitais que conseguiram atingir a meta de $80 \%$ para realização em algum momento da vida (Figura 2a) e sete para os últimos três anos (Figura 2b). 
Médias e proporções de características da população de mulheres entre 25 e 69 anos das capitais brasileiras e do Distrito Federal, segundo o VIGITEL, 2011.

\begin{tabular}{|c|c|c|c|c|}
\hline Capital & $\begin{array}{c}\text { Idade } \\
\text { Média (IC95\%) }\end{array}$ & $\begin{array}{l}\text { Anos de estudos } \\
\text { Média (IC95\%) }\end{array}$ & $\begin{array}{l}\text { Solteiras } \\
\%(I C 95 \%)\end{array}$ & $\begin{array}{l}\text { Tabagismo } \\
\% \text { (IC95\%) }\end{array}$ \\
\hline Aracaju & $48(46-51)$ & $10(9-10)$ & $44(41-47)$ & $8(7-10)$ \\
\hline Belém & $50(47-53)$ & $9(9-10)$ & $44(41-47)$ & $11(9-13)$ \\
\hline Belo Horizonte & $49(46-51)$ & $10(10-10)$ & $42(39-45)$ & $15(13-16)$ \\
\hline Boa Vista & $47(44-51)$ & $9(9-10)$ & $44(41-48)$ & $11(9-14)$ \\
\hline Campo Grande & $49(47-52)$ & $9(9-10)$ & $34(32-37)$ & $13(11-14)$ \\
\hline Cuiabá & $48(46-51)$ & $10(10-10)$ & $38(35-41)$ & $13(11-15)$ \\
\hline Curitiba & $53(51-55)$ & $10(10-11)$ & $33(31-36)$ & $17(15-19)$ \\
\hline Florianópolis & $50(47-52)$ & $11(11-12)$ & $36(33-38)$ & $13(11-15)$ \\
\hline Fortaleza & $47(43-52)$ & $9(9-9)$ & $43(40-46)$ & $9(8-11)$ \\
\hline Goiânia & $49(47-52)$ & $10(10-10)$ & 36 (34-39) & $10(9-12)$ \\
\hline João Pessoa & $51(48-53)$ & $9(9-10)$ & $42(39-45)$ & $10(8-12)$ \\
\hline Macapá & $41(35-47)$ & $7(7-8)$ & $49(44-54)$ & $11(8-15)$ \\
\hline Maceió & $46(44-49)$ & $9(8-9)$ & $42(39-45)$ & $8(6-10)$ \\
\hline Manaus & $47(45-50)$ & $9(9-10)$ & $45(42-48)$ & $11(9-13)$ \\
\hline Natal & $52(49-55)$ & $9(9-10)$ & $42(40-45)$ & $10(8-12)$ \\
\hline Palmas & 45 (40-49) & $11(10-11)$ & $40(37-43)$ & $11(9-13)$ \\
\hline Porto Alegre & $51(49-54)$ & $11(10-11)$ & $38(35-40)$ & $20(18-22)$ \\
\hline Porto Velho & $45(42-48)$ & $9(9-9)$ & $39(37-42)$ & $15(12-17)$ \\
\hline Recife & $52(50-54)$ & $10(9-10)$ & $45(42-48)$ & $11(9-13)$ \\
\hline Rio Branco & $47(44-50)$ & $9(9-9)$ & $37(34-40)$ & $14(12-16)$ \\
\hline Rio de Janeiro & $51(49-53)$ & $10(10-10)$ & $38(35-40)$ & $13(11-14)$ \\
\hline Salvador & $49(46-51)$ & $10(9-10)$ & $47(44-50)$ & $7(6-9)$ \\
\hline São Luís & $48(45-52)$ & $10(10-10)$ & $52(49-55)$ & $11(8-13)$ \\
\hline São Paulo & $50(48-52)$ & $10(9-10)$ & $36(33-38)$ & $18(16-20)$ \\
\hline Teresina & $49(45-52)$ & $9(9-9)$ & $47(44-50)$ & $12(10-14)$ \\
\hline Vitória & $50(47-52)$ & $11(11-12)$ & $40(38-43)$ & $10(8-11)$ \\
\hline Distrito Federal & $49(47-52)$ & $10(10-11)$ & $42(39-45)$ & $10(8-12)$ \\
\hline
\end{tabular}

VIGITEL: Vigilância de Fatores de Risco e Proteção para Doenças Crônicas por Inquérito Telefônico.

A análise demonstrou forte correlação positiva entre o IDH e as proporções dos exames estudados: $r=0,52$ e 0,66 para realização de mamografia em algum momento da vida e nos últimos dois anos, e $\mathrm{r}=0,66$ e 0,71 para realização do Papanicolaou em algum momento da vida e nos últimos três anos, respectivamente (dados não apresentados em tabelas). Quando estimados os coeficientes de correlação entre o IDH e as demais covariáveis de exposição, também foi identificada forte correlação entre este indicador e a escolaridade média da população e o porcentual de pessoas que se declararam brancas. Portanto, essas covariáveis não foram incluídas nos modelos ajustados de regressão.

Na Tabela 3, estão as análises bruta e ajustadas da associação entre as proporções de realiza- ção de mamografia e exames de Papanicolaou e o IDH.Verificou-se que tanto as proporções de mamografia quanto as de Papanicolaou foram associadas com o IDH em todos os modelos propostos. Houve uma redução na razão de prevalência mais evidente da mamografia realizada nos últimos dois anos e dos testes de Papanicolaou. No modelo final ajustado por idade média, situação conjugal, tabagismo, Índice de Gini (per capita) e cobertura de ESF, a razão de prevalência (RP) para realização de mamografia nos últimos dois anos foi de 1,06 (IC95\%: 1,01-1,10) evidenciando que, a cada mudança em 1 desvio padrão do IDH, aumentava em $6 \%$ a proporção de realização das mamografias. Com relação ao exame de Papanicolaou, a cada aumento em 1 desvio padrão do IDH havia um aumento em $7 \%$ na proporção 
Tabela 2

Indicadores socioeconômicos e de acesso ao serviço de saúde das capitais brasileiras e do Distrito Federal, 2011.

\begin{tabular}{|c|c|c|c|}
\hline Cidade & $\mathrm{IDH}$ * & Índice de Gini * & Cobertura da ESF $(\%)$ ** \\
\hline Aracaju & 0,770 & 0,58 & 80 \\
\hline Belém & 0,746 & 0,61 & 17 \\
\hline Belo Horizonte & 0,810 & 0,60 & 76 \\
\hline Boa Vista & 0,752 & 0,58 & 47 \\
\hline Campo Grande & 0,784 & 0,56 & 27 \\
\hline Cuiabá & 0,785 & 0,59 & 39 \\
\hline Curitiba & 0,823 & 0,55 & 33 \\
\hline Florianópolis & 0,847 & 0,54 & 82 \\
\hline Fortaleza & 0,754 & 0,61 & 37 \\
\hline Goiânia & 0,799 & 0,58 & 40 \\
\hline João Pessoa & 0,763 & 0,62 & 76 \\
\hline Macapá & 0,733 & 0,59 & 53 \\
\hline Maceió & 0,721 & 0,63 & 27 \\
\hline Manaus & 0,737 & 0,61 & 29 \\
\hline Natal & 0,763 & 0,61 & 28 \\
\hline Palmas & 0,788 & 0,58 & 68 \\
\hline Porto Alegre & 0,805 & 0,60 & 23 \\
\hline Porto Velho & 0,736 & 0,56 & 61 \\
\hline Recife & 0,772 & 0,68 & 54 \\
\hline Rio Branco & 0,727 & 0,59 & 23 \\
\hline Rio de Janeiro & 0,799 & 0,62 & 23 \\
\hline Salvador & 0,759 & 0,63 & 18 \\
\hline São Luís & 0,768 & 0,61 & 29 \\
\hline São Paulo & 0,805 & 0,62 & 31 \\
\hline Teresina & 0,751 & 0,61 & 98 \\
\hline Vitória & 0,845 & 0,60 & 81 \\
\hline Distrito Federal & 0,824 & 0,63 & 15 \\
\hline
\end{tabular}

ESF: Estratégia Saúde da Família; IDH: Índice de Desenvolvimento Humano.

* Fonte: Instituto de Pesquisa Econômica Aplicada;

** Fonte: Departamento de Informática do SUS.

de realização do exame nos últimos 3 anos ( $\mathrm{RP}=$ 1,07; IC95\%: 1,04-1,10).

\section{Discussão}

Os resultados deste estudo apontam que a proporção de realização de exame de mamografia esteve adequada para grande parte das capitais brasileiras; em contrapartida este resultado não se repetiu para o exame de Papanicolaou. O IDH foi um preditor que apresentou correlação positiva e forte associação com a proporção desses exames realizados, uma vez que a diferença entre o menor e o maior IDH (4 DP) resultou em um acréscimo de $26 \%$ e $31 \%$ na proporção de realização da mamografia nos últimos dois anos e do exame de Papanicolaou nos últimos três anos, respectivamente.

Investimentos na detecção precoce, com cobertura acima de $70 \%$ para mamografia, podem reduzir o número de mortes por câncer de mama em até um terço das mulheres do grupo de risco 19, o que reforça a importância dos resultados encontrados. Outros estudos em nível individual 6,7,20 mostram que cobertura de mamografia nas mulheres do grupo de maior risco para o câncer de mama vem aumentando nas cidades brasileiras. A mulher com baixa renda per capita, com baixa escolaridade, sem plano de saúde privado, sem companheiro, residente em zona rural ou de maior distância do local da mamografia, tem menor chance de realizar o exame ${ }^{4,21}$. $\mathrm{O}$ aumento da cobertura de mamografia por meio da 
Figura 1

Proporção de realização de mamografia (mulheres de 50 a 69 anos) em algum momento da vida e há menos de dois anos, nas capitais brasileiras e Distrito Federal, segundo o VIGITEL, 2011.

1a) Em algum momento da vida

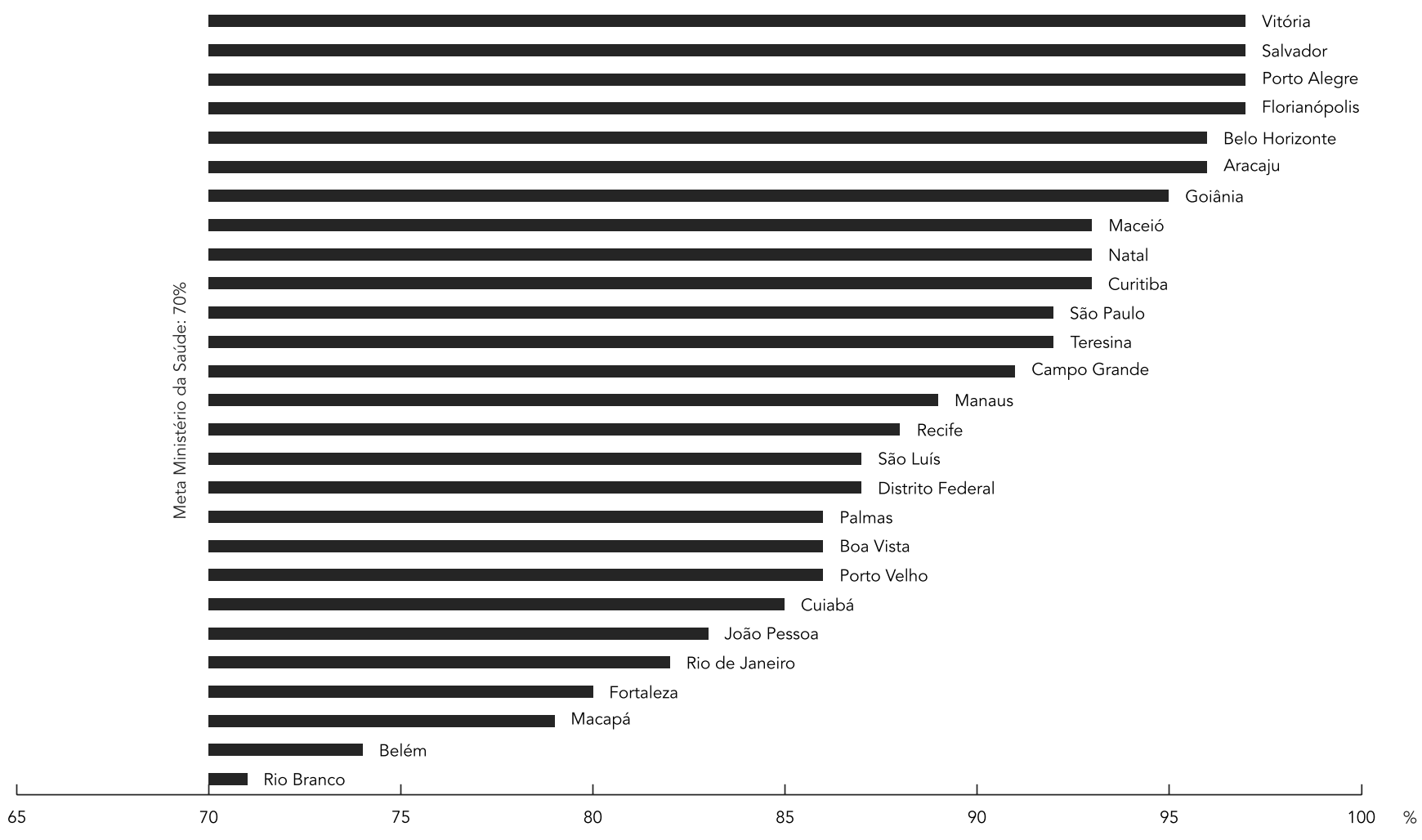

(continua)

redução das iniquidades de renda e escolaridade, fatores intrínsecos ao IDH, foi demonstrado em outro estudo 4 . Mesmo com investimentos do Ministério da Saúde na implantação do programa nacional para melhoria da qualidade da mamografia em 2011 22, permanecem diferenças regionais importantes do acesso até a sua realização 4,23,24. Essas diferenças regionais podem estar relacionadas com as desigualdades sociais existentes entre as regiões, aqui evidenciadas pela associação encontrada entre o IDH e a proporção de realização do exame, mas também pode ser fortemente influenciada por fatores que dizem respeito à utilização dos serviços de saúde, entre os quais estão a oferta dos serviços, a acessibilidade geográfica, a existência de polos de atração e, principalmente, as características de regionalização dos serviços 4,21,25.
Em nossos resultados, os percentuais de cobertura da ESF, cujo programa de atenção à saúde da mulher objetiva a prevenção do câncer de colo uterino e mama, não influenciaram na associação do IDH com a realização da mamografia (Tabela 3). Embora apenas sete capitais $(25,9 \%)$ dentre as vinte e sete unidades da federação possuam cobertura de ESF superior a 65\% (Tabela 2), a requisição do exame também é realizada por profissionais de saúde que atuam no modelo tradicional. A existência de outros modelos de atenção à saúde e a ocorrência de diversas barreiras no âmbito individual ou coletivo na trajetória entre a requisição da mamografia até a sua realização (distância do local de realização ou tempo para agendamento; o grau de esclarecimento e entendimento da população; fatores comportamentais como constrangimento) ${ }^{26}$ poderiam ser fatores justificáveis desse fato. 
Figura 1 (continuação)

1b) Há menos de dois anos

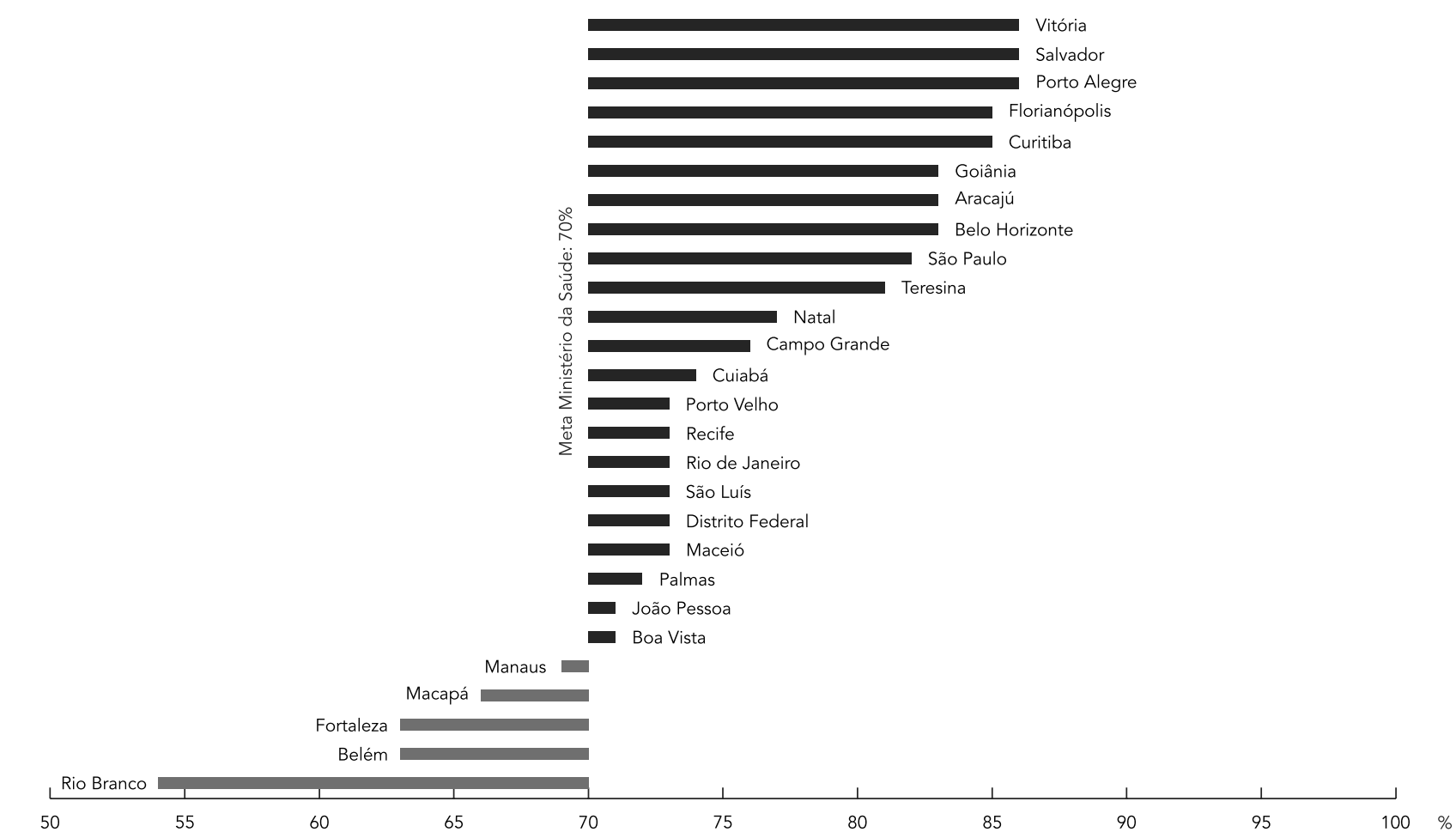

VIGITEL: Vigilância de Fatores de Risco e Proteção para Doenças Crônicas por Inquérito Telefônico.

A Pesquisa Nacional por Amostra de Domicílios, por meio do autorrelato das mulheres, identificou que $71,5 \%$ haviam realizado mamografia nos últimos dois anos 5. Da mesma forma, estudos realizados em cidades brasileiras específicas vêm apresentando resultados consistentes: Juiz de Fora $(72,1 \%)$ 20, Pelotas (70\%) 6 e Maringá $(79 \%)^{7}$. Entre os países de renda alta e de maior IDH que o Brasil, a cobertura de mamografia varia amplamente indo desde $89 \%$ para a Holanda, $86 \%$ para a Finlândia e $72 \%$ para os Estados Unidos até $47 \%$ na França e $24 \%$ no Japão 27. Já a Argentina, um país de renda média-baixa, mas com IDH superior ao do Brasil, tinha uma cobertura de mamografia de 52,6\% em 2009 8. A comparabilidade do IDH e da cobertura de mamografia entre outros países e as capitais brasileiras deve ser avaliada com cautela devido a diferentes níveis de agregação. No entanto, dados de capitais e/ou estados de outros países não estão disponíveis na literatura.
A meta de $80 \%$ para cobertura do exame de Papanicolaou na população de risco 28 aumenta o diagnóstico precoce e pode reduzir em até $90 \%$ a incidência das formas invasivas do câncer de colo do útero 19. Em 2011, o Ministério da Saúde ampliou a faixa etária de rastreamento para 25 a 64 anos 16,22. Neste estudo, a realização do exame nos últimos três anos demonstrou que poucas capitais brasileiras alcançaram o percentual preconizado, não atingindo a cobertura almejada para rastreamento do câncer de colo do útero.

A pesquisa de monitoramento do sistema de saúde brasileiro (2008) identificou que 79,3\% das mulheres brasileiras haviam realizado o exame de Papanicolaou nos últimos três anos 29, e um estudo realizado em 41 municípios do Sul e Nordeste brasileiro encontrou uma prevalência de $75,3 \% 30$. O presente trabalho identificou uma cobertura desse exame semelhante a esses estudos no nível individual (74,3\%). A cobertura de Papanicolaou na Argentina (2009) foi de 60,5\% 8 , nos Estados Unidos (2006) 83\%, no Reino Unido (2006) $80 \%$ e no Canadá (2006) $73 \% 27$. 
Figura 2

Proporção de realização de Papanicolaou (mulheres de 25 a 59 anos) em algum momento da vida e há menos de três anos, nas capitais brasileiras e Distrito Federal, segundo o VIGITEL, 2011.

2a) Em algum momento da vida

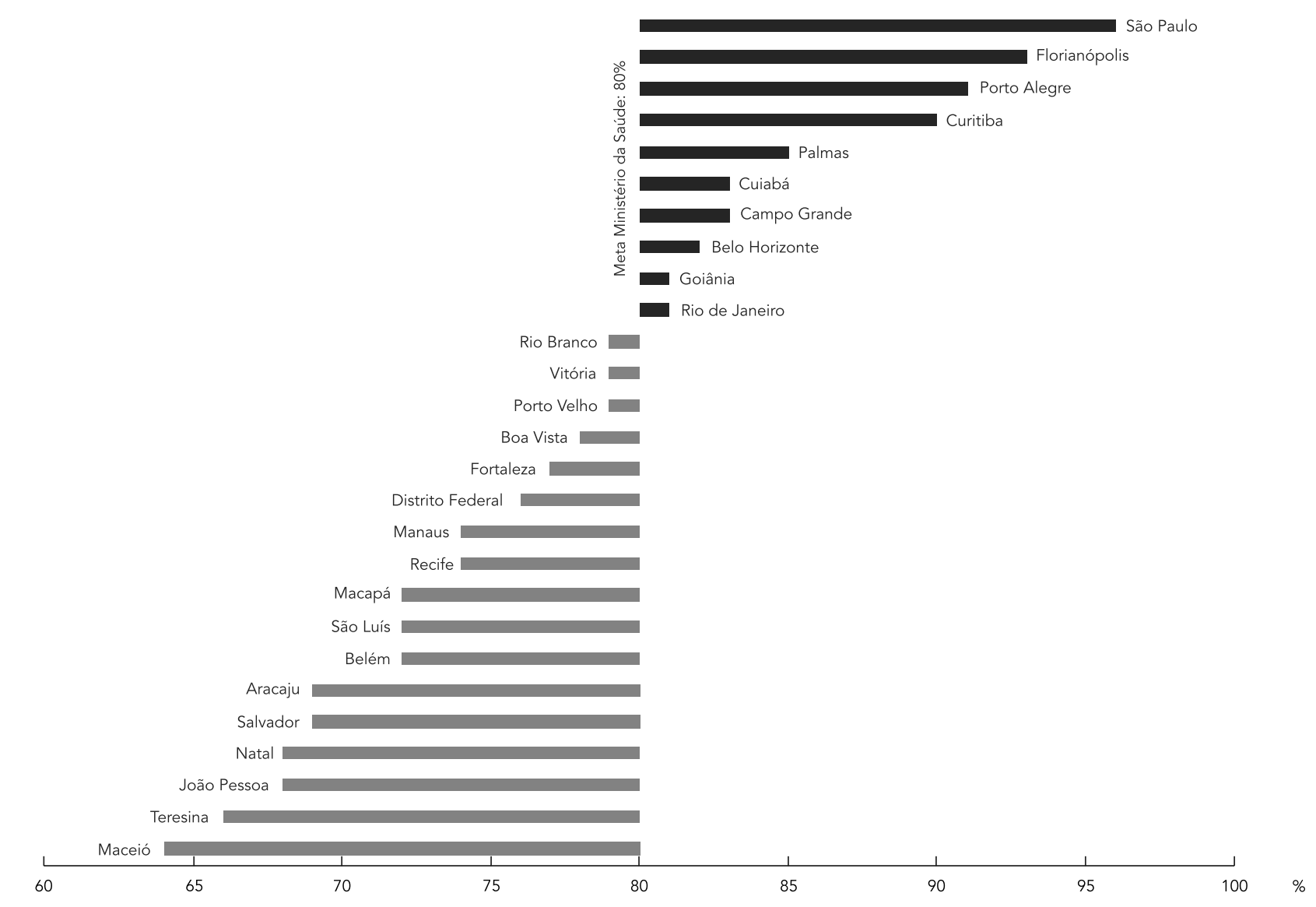

(continua)

Alguns estudos com unidade de análise individual evidenciaram que as metas mínimas preconizadas não vêm sendo atingidas nas cidades com maior vulnerabilidade social e em mulheres com maior risco para o desenvolvimento dessas neoplasias 15,31. Esse fato pode estar associado a um círculo vicioso de dificuldade de acesso à prevenção, rastreamento, ou ainda, intervenção precoce dos processos de saúde/doença com pior desfecho em saúde 21,25. Portanto, enfatiza-se a necessidade de maiores intervenções que deem atenção especial aos grupos sob maior risco, e para que elas sejam efetivas devem incluir medidas que promovam maior equidade no acesso 21 , além de aumentarem a oferta de serviços à população. É importante ressaltar que, municípios do interior do país podem apresentar proporções de realização de mamografia e do exame de Papanicolaou mais baixas, comparando-se com os resultados das capitais apresentados no presente trabalho, uma vez que estes municípios geralmente apresentam menor IDH, maior existência de zonas rurais e menor acesso a serviços específicos como o mamógrafo 4,21, assim como outras diferenças socioeconômicas, que demandariam estratégias específicas facilitadoras para efetividade do rastreamento do câncer de mama e do colo do útero.

Entre os determinantes para a realização da mamografia e do exame de Papanicolaou evidenciados em estudos tendo os indivíduos como unidade de análise, citam-se a idade 4,29, 


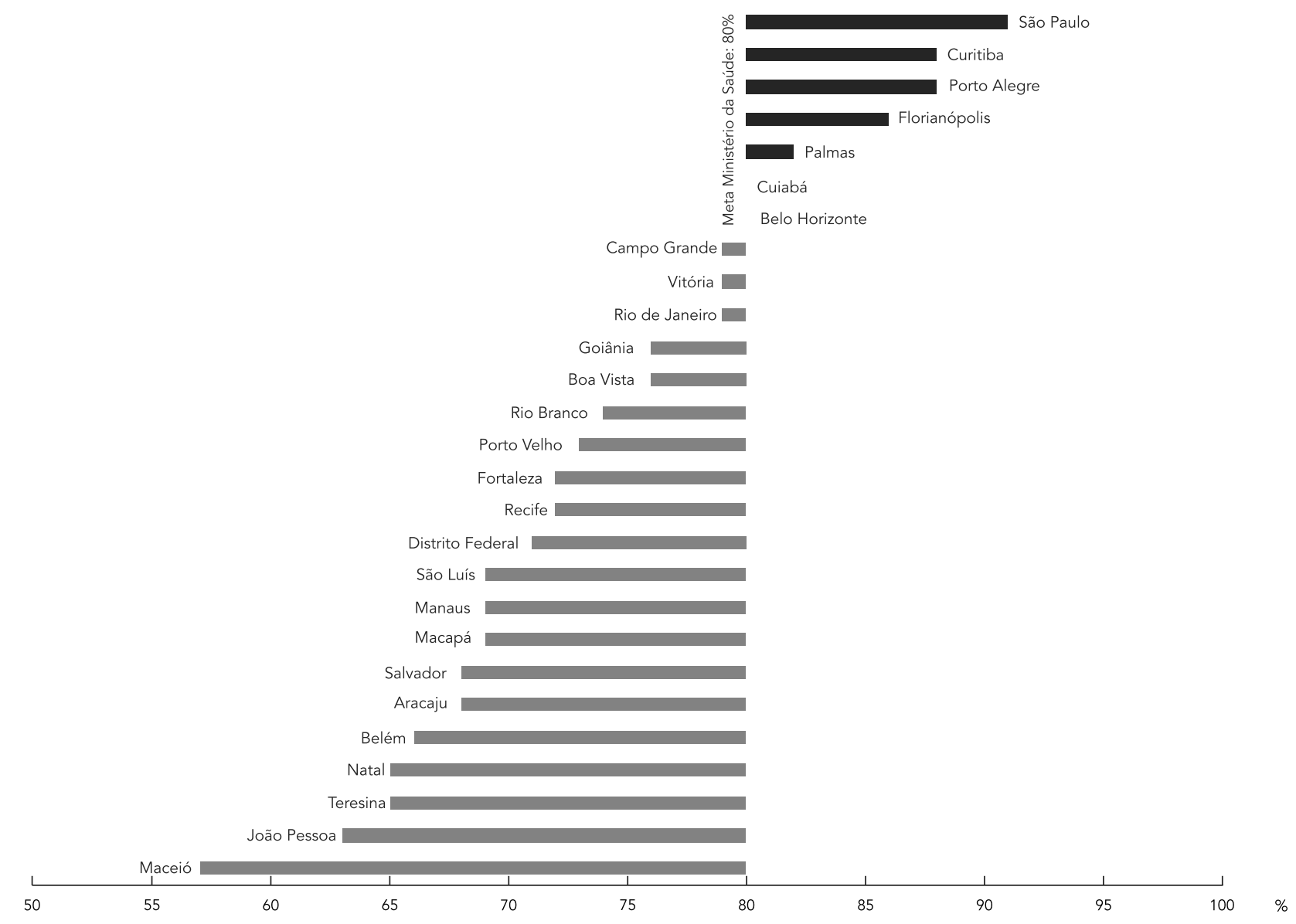

VIGITEL: Vigilância de Fatores de Risco e Proteção para Doenças Crônicas por Inquérito Telefônico.

escolaridade 4,5,7,8,20,23,28,32,33, renda 4,28 , cor da pele 32 , situação conjugal 4,20 , tipo de sistema de saúde utilizado 4 e distância a percorrer para a sua realização 4,21 . Nas análises realizadas no âmbito populacional, embora não tenha sido avaliado o efeito específico de todas esses determinantes na realização dos exames, evidenciou-se que fatores mais comumente descritos não interferiram na associação entre a proporção de exames realizados e o IDH (Tabela 3).

Os fatores culturais e socioeconômicos podem influenciar na busca e na participação da população na realização desses exames. A visita a um médico, por exemplo, foi identificada como o principal determinante socioeconômico para a triagem do câncer de colo do útero num estudo realizado em países da América Latina
34. Entretanto, em cidades com alta cobertura da ESF foram verificadas falhas, sobretudo em alcançar a meta de realização do exame de Papanicolaou, o que reforça a necessidade de um serviço de saúde de qualidade que supere as iniquidades individuais e da coletividade 10. Em nosso trabalho, a inserção da cobertura da ESF no modelo de análise não influenciou o resultado final da associação entre IDH e a realização dos exames nas capitais estudadas. A realização dos exames é influenciada de acordo com formas de financiamento e a participação do setor privado, mas também pelas políticas de organização do sistema de saúde. A realização do exame de Papanicolau, por exemplo, é de competência municipal, sendo possível a coleta do exame nas unidades básicas de saúde, independentemente 
Tabela 3

Análises da associação bruta e ajustadas entre as proporções de mamografia e exame de Papanicolaou * e Índice de Desenvolvimento Humano (IDH) nas capitais brasileiras e no Distrito Federal, segundo o VIGITEL, 2011.

\begin{tabular}{|c|c|c|c|c|}
\hline Indicadores & Bruta & & & \\
\hline & RP (IC95\%) & RP (IC95\%) & RP (IC95\%) & RP (IC95\%) \\
\hline Proporção de mamografia alguma vez na vida & $1,04(1,01-1,07)$ & $1,04(1,01-1,08)$ & $1,04(1,01-1,08)$ & $1,04(1,01-1,07)$ \\
\hline Proporção de mamografia nos últimos 2 anos & $1,07(1,04-1,11)$ & $1,07(1,02-1,12)$ & $1,07(1,02-1,11)$ & $1,06(1,01-1,10)$ \\
\hline Proporção de Papanicolaou alguma vez na vida & $1,07(1,04-1,11)$ & $1,05(1,03-1,07)$ & $1,05(1,03-1,07)$ & $1,06(1,03-1,08)$ \\
\hline Proporção de Papanicolaou nos últimos 3 anos & $1,08(1,05-1,12)$ & $1,07(1,04-1,09)$ & $1,07(1,04-1,09)$ & $1,07(1,04-1,10)$ \\
\hline
\end{tabular}

Ajustada 1: idade média, estado civil e tabagismo; Ajustada 2: idade média, estado civil, tabagismo e Índice de Gini; Ajustada 3: idade média, estado civil, tabagismo, Índice de Gini e cobertura da Estratégia Saúde da Família; RP: razão de prevalência; VIGITEL: Vigilância de Fatores de Risco e Proteção para Doenças Crônicas por Inquérito Telefônico.

* Mulheres de 25 a 59 anos para exame de Papanicolaou e de 50 a 69 anos para mamografia.

da existência de ESF. Por outro lado, a mamografia é de responsabilidade estadual, realizada no nível de atenção secundária, sendo referenciada a outros serviços. Na atenção primária haveria o favorecimento do acesso e utilização por indivíduos de baixa renda, entretanto, para indivíduos de alta renda o acesso seria na atenção secundária 25 . Essas distintas características poderiam gerar diferentes barreiras no acesso e/ou na efetividade da realização desses exames 10,26.

Entre as limitações deste estudo devemos considerar o seu processo de amostragem que selecionou apenas indivíduos usuários de telefonia fixa das capitais brasileiras e Distrito Federal. Atualmente, as pessoas que possuem telefone fixo apresentam maior nível econômico, assim como um melhor acesso ao serviço de saúde e maior probabilidade de realização dos exames avaliados. Portanto, a possibilidade de viés amostral deve ser considerada, mesmo havendo correção para sub ou super-representação de alguns grupos populacionais baseados nos dados do censo do IBGE. A ausência de outros indicadores (como por exemplo, uso da saúde suplementar) ou a existência de inconsistências importantes nos mesmos, impossibilitou que os dados fossem mais bem explorados, o que também pode ser considerada uma limitação deste estudo. Por outro lado, cabe salientar que este estudo foi realizado com base em dados de do- mínio público, cujo caráter periódico de coleta permitirá avaliações futuras das tendências dos indicadores e associações avaliadas. Além disso, a utilização de indicadores referentes à composição sociodemográfica das capitais nos modelos de análise pode ter minimizado a probabilidade de falácia ecológica, uma vez que foram levadas em consideração características reconhecidamente associadas ao nível individual, como idade e situação conjugal.

Portanto, concluímos que a proporção de mulheres, da faixa etária prioritária, que realizaram mamografia nos dois últimos anos esteve dentro do recomendado para a maioria das capitais brasileiras, mas o mesmo não ocorreu para a proporção do exame de Papanicolaou. O IDH padronizado das capitais brasileiras esteve positivamente associado com uma maior proporção desses exames na população. Novas análises que possam avaliar tanto o componente individual quanto o populacional, incluindo fatores comportamentais, seriam de extrema importância para explorar as evidências encontradas. O presente estudo identificou a existência de iniquidade na prevenção secundária do câncer de colo do útero e de mama e a real necessidade de incorporar estratégias que estimulem o desenvolvimento socioeconômico dos municípios compartilhadas por meio das políticas públicas de saúde. 


\section{Resumen}

Estudio ecológico que examinó la proporción de realización de mamografías y citologías, y su asociación con el Índice de Desarrollo Humano (IDH) en 26 capitales de Brasil y el Distrito Federal, en 2011 (datos del VIGITEL). La proporción de mamografía en algún momento de la vida fue superior al 70\% para todas las capitales, y pocas han alcanzado un $80 \%$ en el caso de la citología, en algún momento de la vida, así como en los últimos tres años. Hubo una fuerte correlación positiva entre el IDH $y$ las proporciones de estas pruebas $(r=0,52$ y 0,66 para la realización de la mamografía en algún momento de la vida y los últimos dos años y $r=0,66$ y 0,71 para contar con una citología en algún momento de la vida y en los últimos tres años, respectivamente). La razón de prevalencia de la mamografía en los últimos dos años fue de un 1,06 (IC95\%: 1,01-1,10) y para la citología -en los últimos tres años- fue de un 1,07 (IC95\%: 1,04-1,10). El impulso de políticas públicas que promuevan el desarrollo económico y social de las ciudades brasileñas podría mejorar la cobertura de estos exámenes.

Prueba de Papanicolaou; Mamografía; Desarrollo Humano; Prevención Secundaria

\section{Colaboradores}

Todos os autores colaboraram em todas as etapas do processo.

\section{Referências}

1. Coordenação de Prevenção e Vigilância, Instituto Nacional de Câncer José Alencar Gomes da Silva. Estimativa 2014: incidência de câncer no Brasil. Rio de Janeiro: Instituto Nacional de Câncer José Alencar Gomes da Silva; 2014.

2. World Health Organization. Early detection. Cancer control: knowledge into action. Geneva: World Health Organization; 2007. (WHO Guide for Effective Programmes, Module 3).

3. Secretaria de Gestão Estratégica e Participativa, Ministério da Saúde. Orientações acerca dos indicadores da pactuação de diretrizes, objetivos e metas 2012. Brasília: Ministério da Saúde; 2012.

4. Oliveira EXG, Pinheiro RS, Melo ECP, Carvalho MS Condicionantes socioeconômicos e geográficos do acesso à mamografia no Brasil, 2003-2008. Ciênc Saúde Coletiva 2011; 16:3649-64.

5. Instituto Brasileiro de Geografia e Estatística. Pesquisa Nacional por Amostra de Domicílios. Panorama da saúde do Brasil: acesso e utilização dos serviços, condições de saúde e fatores de risco e proteção à saúde (PNAD, 2008). Rio de Janeiro: Instituto Brasileiro de Geografia e Estatística; 2010.

6. Sclowitz ML, Menezes AM, Gigante DP, Tessaro S. Breast cancer's secondary prevention and associated factors. Rev Saúde Pública 2005; 39:340-9.
7. Matos JC, Pelloso SM, Carvalho MDB. Fatores associados à realização da prevenção secundária do câncer de mama no Município de Maringá, Paraná, Brasil. Cad Saúde Pública 2011; 27:888-98.

8. De Maio FG, Linetzky B, Ferrante D. Changes in the social gradients for Pap smears and mammograms in Argentina: evidence from the 2005 and 2009 National Risk Factor Surveys. Public Health 2012; 126:821-6.

9. Murata IMH, Gabrielloni MC, Schirmer J. Cobertura do Papanicolaou em mulheres de 25 a 59 anos de Maringá - PR, Brasil. Rev Bras Cancerol 2012; 58:409-15.

10. Weissman JS, Schneider EC. Social disparities in cancer: lessons from a multidisciplinary workshop. Cancer Causes Control 2005; 16:71-4.

11. Programa das Nações Unidas para o Desenvolvimento. Valores e desenvolvimento humano 2010 Brasília: Programa de Desenvolvimento das Nações Unidas; 2010.

12. Kiadaliri AA. Social disparity in breast and ovarian cancer incidence in iran, 2003-2009: a time trend province-level study. J Breast Cancer 2013; 16:372-7.

13. Bray F, Jemal A, Grey N, Ferlay J, Forman D. Global cancer transitions according to the Human Development Index (2008-2030): a population-based study. Lancet Oncol 2012; 13:790-801. 
14. Secretaria de Vigilância em Saúde, Ministério da Saúde. Vigitel Brasil 2011: Vigilância de Fatores de Risco e Proteção para Doenças Crônicas por Inquérito Telefônico. Brasília: Ministério da Saúde; 2012.

15. Instituto Nacional de Câncer. Prevenção do câncer de mama. Normas e recomendações do Instituto Nacional de Câncer (INCA). Rev Bras Cancerol 2003; 49:208.

16. Ministério da Saúde. Controle dos cânceres do colo do útero e da mama. 2a Ed. Brasília: Ministério da Saúde; 2013.

17. Battaglia MP, Frankel MR, Link MW. Improving standard poststratification. Techniques for random-digit dialing telephone surveys. Surv Res Methods 2008; 2:11-9.

18. Poole B, Black C, Gelmon K, Kan L. Is Canadian women's breast cancer screening behaviour associated with having a family doctor? Can Fam Physician 2010; 56:e150-7.

19. World Health Organization. National Cancer Control Programmes: policies and managerial guidelines. 2nd Ed. Geneva: World Health Organization; 2002.

20. Novaes CO, Mattos IE. Prevalência e fatores associados a não utilização de mamografia em mulheres idosas. Cad Saúde Pública 2009; 25 Suppl 2:S310-20.

21. Goss PE, Lee BL, Badovinac-Crnjevic T, StrasserWeippl K, Chavarri-Guerra Y, St. Louis J, et al. Planning cancer control in Latin America and the Caribbean. Lancet Oncol 2013; 14:391-436.

22. The Lancet. Initiatives in cancer control form Brazil's Ministry of Health. Lancet Oncol 2013; 14: 385-6.

23. Malta DC, Jorge AO. Análise de tendência de citologia oncótica e mamografia das capitais brasileiras. Ciênc Cult 2014; 66:25-9.

24. Instituto Brasileiro de Geografia e Estatística. Indicadores sociodemográficos e de saúde no Brasil 2009. Rio de Janeiro: Instituto Brasileiro de Geografia e Estatística; 2009.

25. Garcia-Subirats I, Vargas I, Mogollón-Pérez AS, De Paepe P, da Silva MR, Unger JP, et al. Inequities in access to health care in different health systems: a study in municipalities of central Colombia and north-eastern Brazil. Int J Equity Health 2014; 13:10.
26. Glasgow RE, Whitlock EP, Vogt TM. Barriers to mammography and pap smear screening among women who recently had neither, one or both types of screening. Ann Behav Med 2000; 22:223-8.

27. Organization for Economic Co-operation and Development. Health at a Glance 2009: OECD indicators. Paris: OECD Publishing; 2009.

28. Instituto Nacional de Câncer. Normas e recomendações do INCA: periodicidade de realização do exame preventivo do câncer do colo do útero. Rev Bras Cancerol 2002; 48:13-5.

29. Laboratório de Informações em Saúde, Instituto de Comunicação e Informação em Ciência e Tecnologia, Fundação Oswaldo Cruz. PROADESS Avaliação de Desempenho do Sistema de Saúde Brasileiro: indicadores para monitoramento. Relatório. Rio de Janeiro: Fundação Oswaldo Cruz; 2008.

30. Correa MS, Silveira DS, Siqueira FV, Facchini LA, Piccini RX, Thumé E, et al. Pap test coverage and adequacy in the South and Northeast of Brazil. Cad Saúde Pública 2012; 28:2257-66.

31. Martins LFL, Thuler LCS, Valente JG. Cobertura do exame de Papanicolaou no Brasil e seus fatores determinantes: uma revisão sistemática da literatura. Rev Bras Ginecol Obstet 2005; 27:485-92.

32. Hackenhaar AA, Cesar JA, Domingues MR. Exame citopatológico de colo uterino em mulheres com idade entre 20 e 59 anos em Pelotas, RS: prevalência, foco e fatores associados à sua não realização. Rev Bras Epidemiol 2006; 9:103-11.

33. Chor D, Oliveira EXG, Melo ECP, Pinheiro RS, Carvalho MS. Desigualdade socioeconômica afeta a chance de realizar mamografia no Brasil. http:// cmdss2011.org/site/wp-content/uploads/2011/10/ CMDSS-1110.1v1.pdf.

34. Soneji S, Fukui N. Socioeconomic determinants of cervical cancer screening in Latin America. Rev Panam Salud Pública 2013; 33:174-82.

Recebido em 08/Mai/2014

Versão final reapresentada em 13/Dez/2014 Aprovado em 09/Jan/2015 